\title{
Real-time measurement of Plasmodium falciparum-infected erythrocyte cytoadhesion with a quartz crystal microbalance
}

\author{
Daniela Kömpf ${ }^{1,4 \dagger}$, Jana Held ${ }^{2,3^{*}}$, Stefani F. Müller ${ }^{1}$, Hartmut R. Drechsel ${ }^{1,5}$, Serena C. Tschann ${ }^{2,3}$, \\ Hinnak Northoff' ${ }^{1}$, Benjamin Mordmüller ${ }^{2,3}$ and Frank K. Gehring ${ }^{1,5^{*}}$
}

\begin{abstract}
Background: An important virulence mechanism of the malaria parasite Plasmodium falciparum is cytoadhesion, the binding of infected erythrocytes to endothelial cells in the second half of asexual blood stage development. Conventional methods to investigate adhesion of infected erythrocytes are mostly performed under static conditions, many are based on manual or semi-automated read-outs and are, therefore, difficult to standardize. Quartz crystal microbalances (QCM) are sensitive to nanogram-scale changes in mass and biomechanical properties and are increasingly used in biomedical research. Here, the ability of QCM is explored to measure binding of P. falciparum-infected erythrocytes to two receptors: CD36 and chondroitin sulfate A (CSA) under flow conditions.
\end{abstract}

Methods: Binding of late stage P. falciparum parasites is measured in comparison to uninfected erythrocytes to CD36- and CSA-coated quartzes by QCM observing frequency shifts. CD36-expressing cell membrane fragments and CSA polysaccharide were coated via poly-L-lysine to the quartz. The method was validated by microscopic counting of attached parasites and of erythrocytes to the coated quartzes.

Results: Frequency shifts indicating binding of infected erythrocytes could be observed for both receptors CD36 and CSA. The frequency shifts seen for infected and uninfected erythrocytes were strongly correlated to the microscopically counted numbers of attached cells.

Conclusions: In this proof-of-concept experiment it is shown that QCM is a promising tool to measure binding kinetics and specificity of ligand-receptor interactions using viable, parasite-infected erythrocytes. The method can improve the understanding of the virulence of $P$. falciparum and might be used to cross-validate other methods.

Keywords: Malaria, PfEMP1, Cytoadhesion, Thickness shear mode sensors, Biosensor, CSA, CD36

\section{Background}

Quartz crystal microbalances (QCM) are highly sensitive sensors that can reliably weigh material in the nanogram range and are highly sensitive to changes in biomechanical properties of coupled biomaterial. They consist of a

\footnotetext{
*Correspondence: janaheld@hotmail.de; fkg.gehring@gmail.com ${ }^{\dagger}$ Daniela Kömpf and Jana Held contributed equally to this work ${ }^{1}$ Biosensor Research Group, Institute of Clinical and Experimental Transfusion Medicine, University Hospital of Tübingen, Tübingen, Germany

${ }^{2}$ Institute of Tropical Medicine, University of Tübingen, Tübingen, Germany

Full list of author information is available at the end of the article
}

thin, usually round slice of crystalline quartz with a gold electrode on each side. Oscillating crystals are composed of $\alpha$-Quartz $\left(\mathrm{SiO}_{2}\right)$ that is known to be piezoelectric material, characterized by: (a) appearance of electrical potential when it is subjected to mechanical stress (piezoelectric effect); and, (b) deformation of the material when it is subjected to electrical potential (inverse piezoelectric effect). Due to the piezoelectric character the quartz is stimulated to oscillations when connected to $A C$ voltage [1]. Material attaching to the quartz's surface reduces the frequency. Thus, an increase in weight leads to an adequate decrease in frequency. In biosciences, mass-sensitive QCM are already well accepted, while 
applications for clinical questions, in particular with whole blood samples, are novel and have not yet been exploited. Lately, this technology has been applied to the typing of blood groups, detection of bacteria, bacterial toxins, viruses, and measuring affinity of antibodies. Further studies on adherent cell types have been performed but the measurement of whole cell interactions of primarily non-adherent cells has not been addressed thoroughly [1-6].

Here a novel QCM-based assay is presented to evaluate whole cell interactions by measuring cytoadhesion of erythrocytes infected with the malaria parasite Plasmodium falciparum to endothelial cell receptors. Malaria is the most important parasitic infection and accounted for more than 200 million episodes and 438,000 deaths in 2015 [7]. The disease is caused as a result of the infection by protozoan parasites of the genus Plasmodium. Symptoms only occur during the blood stage of the infection when parasites multiply in red blood cells. Among five different species known to infect humans, $P$. falciparum causes the most severe form of the disease and is responsible for the vast majority of deaths. In contrast to the other species, $P$. falciparum-infected red blood cells (iRBCs) adhere to vascular endothelium of postcapillary venules (sequestration) and to non-infected erythrocytes (rosette formation) in the second half of their asexual replication, cycle via $P$. falciparum erythrocyte membrane protein 1 (PfEMP1), which is expressed on the surface of iRBCs $[8,9]$. Through cytoadhesion the parasite avoids clearance by the spleen; this is an immune evasion mechanism that can lead to excessive parasite multiplication and may result in complications such as cerebral and placental malaria [10-13]. Important receptors involved in this process are CD36, ICAM1 in cerebral malaria and chondroitin sulfate A (CSA) in placental malaria [1416]. This work focuses on the binding of iRBC to CD36 and CSA. CD36 is expected to be the main receptor of iRBC adhesion as nearly all laboratory isolates of $P$. falciparum, as well as clinical isolates from patients attach to this receptor [15-17]. CSA on the other hand, is well described to be the main receptor of parasite binding in placental malaria and parasites expressing the highly conserved PfEMP1 gene var2csa are known to bind to this polysaccharide $[14,18,19]$. Placental malaria can cause low birth weight of newborns, premature delivery, abortion, stillbirth, maternal anaemia, as well as infant and maternal morbidity and mortality [20,21]. CSA is also present on other endothelial tissue where parasites might bind to it [22].

Different methods have been developed to measure PfEMP1-mediated cytoadhesion. Up until now most of the established cytoadhesion assays have been performed under static conditions [23-26], thus lacking shear stress which is assumed to be important for the parasites to get in contact with the appropriate receptors. However, flowbased assays exist as well and some include static periods at the beginning of the measurement to allow settling of parasites, so that they are able to make contact with the immobilized receptors [27-32]. The disadvantage of some of these systems is that handling of the constructions is cumbersome; for example, the read-out is microscopy-based and therefore analysis of results is less standardized [33-36]. Hence, a real-time detection of cytoadhesion is not possible and an adequate evaluation of the experiments performed with these microscopebased assays is time consuming and often reader biased. Another limiting aspect is that measuring of binding times and kinetics as well as discrimination between loosely adsorbed and truly adhered cells are very difficult.

The analysis of this complex interaction would benefit from other highly sensitive and reproducible methods based on a different technology for validation. Therefore, QCM is adapted in this proof-of-concept experiment for the analysis of PfEMP1-mediated iRBC binding to the cell surface of CD36-expressing melanoma cells as well as to CSA. It is the first time that QCM under flow conditions has been used with viable iRBCs and these experiments show the great potential of this approach for future studies in cell-cell as well as cell-receptor studies.

\section{Methods \\ Parasite culture}

The parasite strains FCR3-CD36 and FCR3-CSA were kindly provided by Artur Scherf, Institute Pasteur Paris, and were cultivated as previously described [37] in fresh group O+ erythrocytes at $5 \%$ haematocrit in RPMI-1640 supplemented with $2 \mathrm{mM}$ L-glutamine, $50 \mu \mathrm{g} / \mathrm{ml}$ gentamycin, $25 \mathrm{mM}$ HEPES, $0.5 \%$ Albumax II, and $5 \% \mathrm{AB}+$ human serum (complete medium). Culture flasks were aerated with $5 \%$ carbon dioxide, $5 \%$ oxygen and $90 \%$ nitrogen. Specific binding phenotype of parasites was maintained by regular selection. For CD36 this was performed by monthly panning of late-stage, infected erythrocytes on C32-melanoma cells, at $\mathrm{pH} 6.8$, as described elsewhere [38, 39]. To select parasites for specific binding to CSA, late blood stages were panned monthly on polystyrene six-well plates, coated with CSA from bovine trachea (Sigma) as previously described [40]. No negative selection of parasites was done and parasites were used for experiments after at least one round of selection. Prior to adhesion assays, cultures were synchronized by treatment with $5 \%$ sorbitol and late stages were purified/ enriched by magnetic cell sorting (MACS) as described elsewhere [41]. 


\section{C32-melanoma cell culture}

C32-melanoma cells expressing large amounts of CD36 were obtained from the American Type Culture Collection (ATCC) and cultured at $5 \%$ carbon dioxide and $37{ }^{\circ} \mathrm{C}$ using DMEM supplemented with $50 \mu \mathrm{g} / \mathrm{ml}$ gentamycin, 10 \% FCS, 2 mM L-glutamine, and $1 \%$ MEM non-essential amino acid solution (NEAA). Splitting of cells was performed when cultures reached 80-90\% confluence.

\section{QCM sensors}

The QCM sensors used in this work were AT-cut, $10 \mathrm{MHz}$ quartz crystals $(8 \mathrm{~mm}$ in diameter, thickness of $166 \mu \mathrm{m}$ (KVG Quartz Crystal Technology, Neckarbischofsheim, Germany) with a special gold electrode design developed by FKG.

\section{Base coating of quartz surface}

To successfully immobilize receptors to the quartz, a base coating with poly-L-lysine (PLL, Fluka $70-150 \mathrm{kDa}$ ) to untreated sensors was done by dropping $60 \mu \mathrm{l}$ of $0.5 \mathrm{mg} /$ $\mathrm{ml}$ PLL in $\mathrm{H}_{2} \mathrm{O}$ to the quartz and subsequent drying under a stream of nitrogen [25]. For each experiment a new quartz was used as a complete cleaning of used quartzes was not possible.

\section{Coating of $\mathrm{CD} 36$ to the quartz surface}

CD36 belongs to the class B scavenger receptor proteins and is characterized by a special structure that is necessary to ideally present the specific binding site to parasitized erythrocytes. Thus, to preserve this structure for CD36 coating, membranes or membrane fragments of C32 cells were prepared by homogenization of C32 cells with a potter on ice using a fractioning buffer consisting of Tris/ $\mathrm{HCl}(50 \mathrm{mM}), \mathrm{NaCl}(150 \mathrm{mM})$ and EDTA $(1 \mathrm{mM})$. To separate the membrane fragments from nuclei and intact cells, the suspension was centrifuged at $540 \times g\left(10 \mathrm{~min}, 4{ }^{\circ} \mathrm{C}\right)$. In a next step the supernatant was centrifuged at $2500 \times g\left(15 \mathrm{~min}, 4{ }^{\circ} \mathrm{C}\right)$ to remove further organelles. The last centrifugation step using an ultracentrifuge at $100,000 \times g\left(1 \mathrm{~h}, 4{ }^{\circ} \mathrm{C}\right)$ served to concentrate the membrane fragments. After removal of the supernatant the pellet was re-suspended in $400 \mu \mathrm{l}$ fractioning buffer supplemented with $800 \mu \mathrm{l}$ of a proteinase-inhibitor solution (Roche). Aliquots were stored at $-20^{\circ} \mathrm{C}$. To achieve a coating as homogeneous as possible, membrane suspensions were incubated with PBS-Tween-20 $(0.1 \%)$ for 5 min. Immobilization on PLL-coated quartzes was done by dropping $60 \mu \mathrm{l}$ of suspension to the quartz, and subsequent incubation for $10 \mathrm{~min}$ before unbound membranes were washed away with PBS. This was followed by fixation with $4 \%$ paraformaldehyde in PBS on ice for $7 \mathrm{~min}$ and blocking with $2 \%$ BSA in PBS (1 h, RT).

\section{Coating of CSA to the quartz surface}

To coat CSA to the quartz surface CSA (Sigma, $1 \mathrm{mg} / \mathrm{ml}$ in PBS) was immobilized 'online' to PLL. Following the insertion of the PLL-coated quartz to the QCM platform, the CSA solution was allowed to run over the quartz at a flow rate of $50 \mu \mathrm{l} / \mathrm{min}$ for $10 \mathrm{~min}$ to enable CSA to bind to the PLL-coated quartz. This was followed by a washing step with flow medium for $5 \mathrm{~min}$ at $100 \mu \mathrm{l} / \mathrm{min}$.

To verify binding specificity for PLL basis coating, control human serum was added to the quartz outside of the platform before adding iRBCs (FCR3-CSA) without prior coating of CSA.

\section{Verification of coating by immunofluorescence}

Distribution of the receptors CD36 and CSA on the quartz were verified by an indirect immunofluorescence test (IFT). A separate sample was treated with secondary antibody only, to control for unspecific binding of the antibody. After coating, quartzes were fixed with $4 \%$ paraformaldehyde in PBS on ice for 7 min, followed by blocking with $2 \%$ BSA in PBS ( $1 \mathrm{~h}, \mathrm{RT})$.

The distribution of CD36 on the membrane of C32 cells was visualized by staining with a monoclonal mouseanti CD36 antibody (Abcam, 1:10 in PBS, $1 \mathrm{~h}, \mathrm{RT}$ ); and a sheep anti-mouse IgG $\mathrm{F}\left(\mathrm{ab}^{\prime}\right) 2$ fragment-FITC antibody (Sigma, 1:30 in PBS, 1 h, RT). For verification of CSAcoating, quartzes were stained with a monoclonal mouse anti-CSA antibody (Sigma, 1:100 in PBS, $1 \mathrm{~h}, \mathrm{RT}$ ) and a FITC-labelled goat anti-mouse IgM antibody (Sigma, 1:128, 1 h, RT).

Pictures of stained quartzes were taken by the digital camera Casio QV 5700 at the Axioskop 2MAT microscope with $100 \times$ or $500 \times$ magnification. Overlay of pictures was made with Adobe Photoshop 7.0.

\section{Sensor platform and adhesion assay using QCM-sensors}

QCM-sensors can detect very low mass changes deposited on the quartz by changes in resonance frequency. Frequency shifts due to adhesion processes to receptors were electronically recorded and analysed using a sensor platform called 'Fidget Type 1' [42, 43] (Fig. 1a). As two integrated QCM sensor channels exist, it is possible to simultaneously examine two samples (sample + control) under the same conditions. The platform is equipped with an automated flow-injection system and a Peltier element, which enables stable measurements at specific temperatures. Control measurements with human serum focusing on the PLL coating systems were performed at a later time point on the further developed and commercially available beta version sensor platform qCell $\mathrm{T}$ [44] (Fig. 1b). The one channel platform qCell T provides dissipation measurements in addition to the frequency curves. However, as dissipation curves do not 


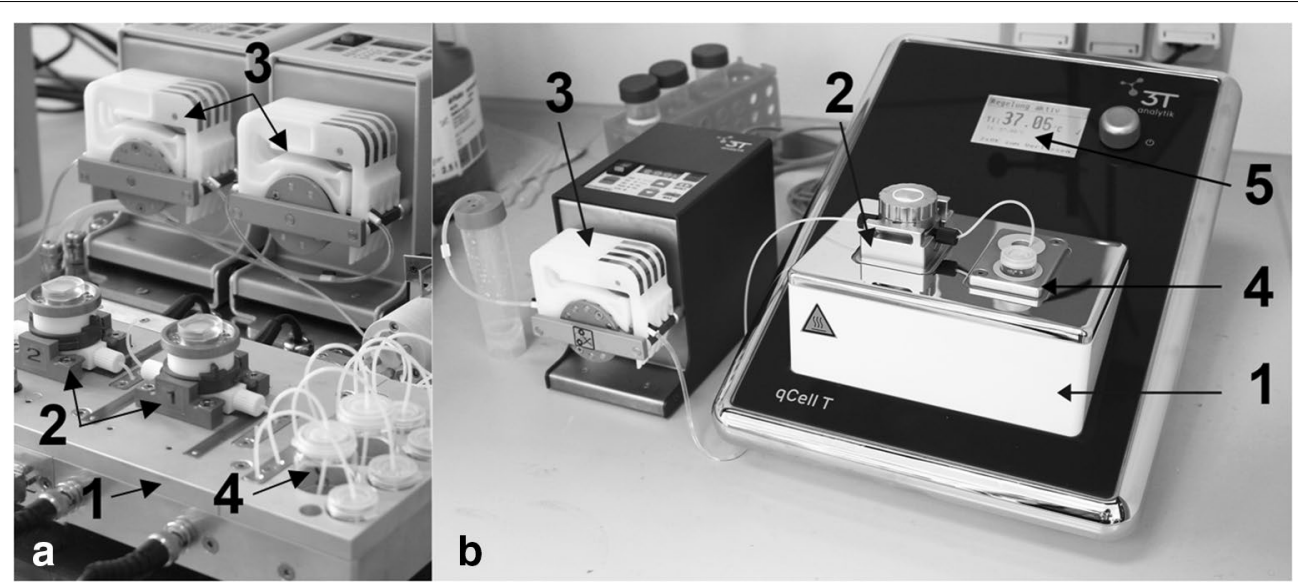

Fig. 1 QCM platform settings. a Fidget Type 1 and $\mathbf{b}$ qCell T. Thermo-controlled QCM platform (1) with fluidic measuring chambers for QCM-sensors (2); peristaltic pumps (3); fluidic system with sample retainers (4); and, digital display (5)

add substantial information to the results they are not presented here. The other functions and results obtained from the measurements are comparable to the old version but stability, sensor handling and software have been essentially improved.

To ensure a sterile environment for measurements, the fluidic system was first disinfected by incubation with $1 \%$ sodium hypochlorite for $10 \mathrm{~min}$. After washing with sterile distilled water and a de-gassed flow medium (complete medium without sodium hydrogen carbonate and without human serum), sensors were inserted into the pre-heated platform $\left(37^{\circ} \mathrm{C}\right)$. Cell suspensions were diluted in flow medium and were transferred to the sensor surface at a continuous flow rate $(50 \mu \mathrm{l} / \mathrm{min})$.

The optimal parameters for detection of adhesion processes to the receptors by iRBCs were achieved by systematically adapting measurement periods, cell concentrations and flow rates. Final experiments with CD36 and CSA coated to PLL were performed with the optimized closed fluidics system. The flow rate was adjusted to $50 \mu \mathrm{l} / \mathrm{min}$ to fill the fluidic system at the onset of the measurement with flow medium, and to pump the cell suspension over the sensor during the period of the whole measurement of cell adhesion. For the assays, $1.5 \times 10^{5} \mathrm{iRBCs} / \mathrm{ml}$ or $\mathrm{RBCs} / \mathrm{ml}$ were diluted in flow medium and allowed to circulate and flow over the quartz at $50 \mu \mathrm{l} / \mathrm{min}$ for $2.5 \mathrm{~h}$ in a closed system. The inner diameter of the tubes used was $0.38 \mathrm{~mm}$, resulting in a velocity of $73 \mathrm{~mm} / \mathrm{s}$ for the used flow rate.

Verification of parasite adhesion by microscopy after QCM After binding experiments, quartzes were removed, rinsed with flow medium and fixed with $4 \%$ paraformaldehyde in PBS on ice for $7 \mathrm{~min}$. To determine the stage and viability of iRBCs adherent to the sensor's surface, samples were stained with the DNA-stain DAPI (Sigma, $0.2 \mu \mathrm{g} / \mathrm{ml}, 5 \mathrm{~min}, \mathrm{RT})$. The mean number of bound iRBCs and RBCs of three visual fields, corresponding to a surface of $22.1 \times 10^{3} \mu \mathrm{m}^{2}$, were determined by counting under a microscope (AxioImager, Zeiss, magnification $500 \times)$.

\section{Analysis}

Frequency shifts were analysed using qCell $\mathrm{T}$ software. Median differences, the interquartile range as well as the single measurements are shown for frequency shifts and the subsequent microscopic count data of attached parasites to the quartz. Microscopic counts of attached infected and uninfected RBCs are correlated to frequency shifts by Spearmans rho for paired samples.

\section{Results}

\section{Verification of CD36 coating of the quartz}

Expression of CD36 on C32 melanoma cells was verified by fluorescence microscopy, after staining with an anti-CD36 antibody showing an intense staining evenly spread over the surface of the cell (Fig. 2a). Isolated cell membranes of melanoma cells were immobilized to the quartz surface via PLL and were stained with an antiCD36 antibody.

Images obtained by fluorescence microscopy show that an almost homogenous coating of the quartzes with CD36 could be achieved and that the CD36 antibody epitope was not destroyed by the processes of cell homogenization and the following differential centrifugation (Fig. 2b). Minor differences in the dissemination of membranes to the sensors are caused by the nature of the biological medium in which CD36 is embedded. 


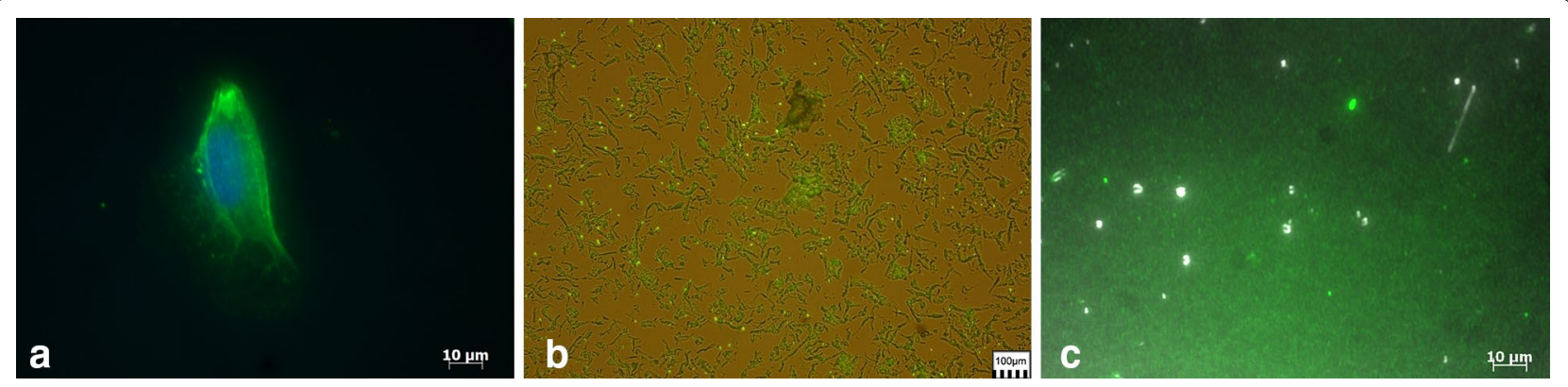

Fig. 2 Quartz coating with CD36 using membranes of melanoma cells and CSA via PLL. a Overlay picture of a C32 melanoma cell stained for DNA (DAPI, blue) and CD36 (FITC, green) showing a homogenous spread of CD36 on the surface and the nucleus in the centre of the cell; $\mathbf{b}$ fluorescence picture of isolated and homogenized cell membranes of C32 melanoma cells immobilized to a PLL-coated quartz and stained with a CD36 antibody (FITC, green); c overlay picture of a quartz coated with PLL/CSA showing an even distribution of fluorescence signal, indicating a homogenous coating of CSA to the quartz. The quartz was stained with an anti-CSA antibody (FITC, green)

To preserve the complex structure of the CD36 protein, which belongs to class B scavenger receptor proteins, whole membranes of C32 melanoma cells are used. Whole cells could not be used as the layer on the sensor would be too thick and not rigid enough to determine interactions of the parasites with the coated sensors.

\section{Verification of CSA coating via fluorescence microscopy}

Coating of the quartz sensor with the receptor was confirmed by fluorescence microscopy (Fig. 2c). CSA immobilized via PLL resulted in a homogenous and dense coating, illustrated by a strong, specific fluorescence signal. Coating of the sensor with CSA via PLL was additionally verified by on-line measurement in the platform. Injection of CSA led to a strong binding signal, which remained stable even after rinsing with high flow rates. Further incubation steps with different concentrations of bovine serum albumin (BSA) showed no additional binding events (Additional file 1). Thus, potential unspecific binding sites appear to be non-accessible.

\section{Measurement of binding of Plasmodium falciparum iRBCs to receptors (CD36 and CSA) via QCM}

In Fig. 3 an overview of the frequency shifts reflecting the mass change due to binding is shown in comparison to the microscopic counts of attached cells on the quartz. Parasites selected for CD36 binding (FCR3-CD36) adhered to CD36, reflected by a dampening of the signal (median frequency shift: $-187 \mathrm{~Hz}, \mathrm{n}=6$ ), whereas uninfected RBCs did not adhere to CD36 (median frequency shift: $-58.5 \mathrm{~Hz}, \mathrm{n}=4$ ). This was also reflected by the microscopic cell count showing that a median of 36 iRBCs $(n=6)$ attached to one visual field of the CD36-coated quartz, whereas only a median of 1.5 RBCs $(\mathrm{n}=4)$ attached in the control experiment. The parasite strain selected for CSA binding (FCR3-CSA), specifically adhered to CSA immobilized to PLL, reflected by a dampening of the signal (median frequency shift: $-9 \mathrm{~Hz}$, $\mathrm{n}=5$ ) after adding of parasites to the flow system. Uninfected RBCs did not bind (median frequency shift: $133 \mathrm{~Hz}, \mathrm{n}=3$ ). The results are reflected by the parasite counts performed after the experiments. When checking for attached parasites a median of 14 iRBCs $(n=5)$ could be counted in one visual field per quartz compared to a median of 0 attached RBCs $(n=3)$ on the control quartz. For the measurement of RBCs on the CSA-coated quartz, an increase in frequency occurred which is caused by a so-called 'missing mass effect' [45] leading to alterations in visco-elasticity.

Addition of late stage iRBCs to CD36 and late stage iRBCs to CSA led to a strong frequency shift compared to RBCs only. Representative frequency shifts obtained with infected and uninfected RBCs for a PLL-CD36-coated quartz are shown in Fig. 4a and for a PLL-CSA-coated quartz in Fig. 5a. Addition of infected or uninfected RBCs led to a short peak due to stopping of the pumps at the beginning of the measurement. Starting and stopping of the pumps led to differences in frequencies that restored when the normal flow rate $(50 \mu \mathrm{l} / \mathrm{min})$ was applied. After the short peak observed at the beginning of the measurement, a decrease in frequency appeared caused by the cells arriving on the sensor's surface. Thus, the sensors detected the occurring mass change. The continuous flow of medium over the sensor rapidly spread the cells over the sensor and unbound cells were washed away leading to a balance of the frequency $(\leq 5 \mathrm{~min})$. The slow decrease of frequency over the $2.5 \mathrm{~h}$ was due to the continuous adhesion process of parasites during the experiment. When adding RBCs, the signal stayed at the same level or even increased. The increase for RBCs seen in Fig. 5a was due to the visco-elastic behaviour of the PLL/ CSA layer, caused by the missing mass effect, mentioned 


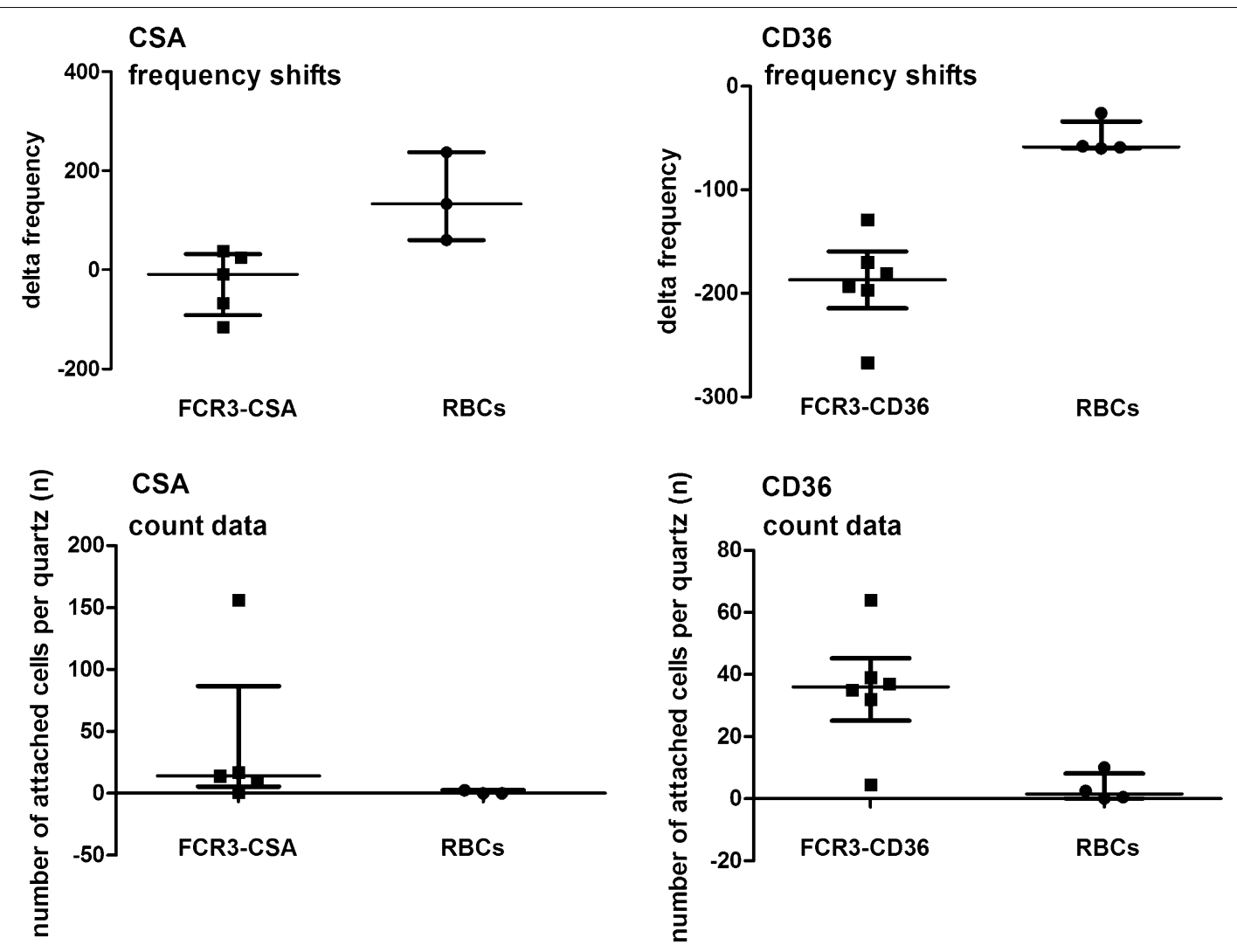

Fig. 3 Overview of QCM experiments measuring attachment of cells to receptors by frequency shifts and subsequent microscopic count. Median, interquartile range and single measurements of frequency shifts measured by the QCM platform Fidget Type 1 (upper panel) and subsequent microscopic count of the number of attached cells per microscopic field (lower panel) for either P. falciparum iRBCs of FCR3-CSA strain $(n=5)$ and red blood cells (RBC, $n=3$ ) added to CSA receptors (CSA), or for iRBCs of FCR3-CD36 strain $(n=6)$ and RBCs $(n=4)$ added to CD36 receptors. Results show that iRBCS (FCR3 parasites) bind to the respective receptor, reflected by a dampening of the frequency and a higher count data in the microscope whereas RBCs do not bind. CSA chondroitin sulfate A, PLL poly-L-lysin

above. Differences between the course of the two signals of CD36 and CSA are due to the differently coated quartzes; for CD36, whole cell membrane fragments were immobilized to the quartz surface whereas for CSA the quartz was coated with the polysaccharide alone. As it is assumed that iRBCs develop stronger adhesion to CD36 than to CSA, different binding kinetics can be observed.

The number of attached cells was correlated with the observed frequency shift (rho $=-0.55, \mathrm{p}=0.017$ ). Example images of one microscopic field are given in Fig. 4b, c for CD36 and iRBCs and RBCs, respectively, and in Fig. 5b, c for CSA and iRBCs and RBCs, respectively. They show that iRBCs with FCR3-CD36 parasites attached to CD36-coated quartzes and that iRBCS with FCR3-CSA parasites attached to CSA-coated quartzes (Figs. 4b, 5b), in contrast to uninfected RBC (Figs. 4c, 5c), where only a very few cells attached.

PLL by itself is very sticky for any kind of cell and can therefore not be used without a receptor/analyte. PLLcoated quartzes alone bound added cells very strongly and quickly (within minutes) (see Additional file 2 for $\mathrm{RBCs}$ ). As a separate control experiment, human serum proteins were immobilized to PLL and as expected, frequency shifts showed that iRBCs (FCR3-CSA) did not bind to human serum proteins [median frequency shift: $46 \mathrm{~Hz}(\mathrm{n}=3)$, Additional file 3].

\section{Discussion}

Measuring with QCM allows real-time observation of cellular interactions by evaluating adhesion processes of intact $P$. falciparum-infected RBCs and their respective receptor. Here a novel method is presented to measure cell adhesion processes of iRBCs to two different receptors under flow conditions. Binding is analysed of iRBCs to CD36, a receptor most $P$. falciparum isolates adhere to, and binding to CSA, the main receptor involved in placental malaria. Dampening of the signal showed that iRBCs bound with higher affinity to the receptor when compared to uninfected RBCs, corresponding with the results obtained by microscopic counting of attached 

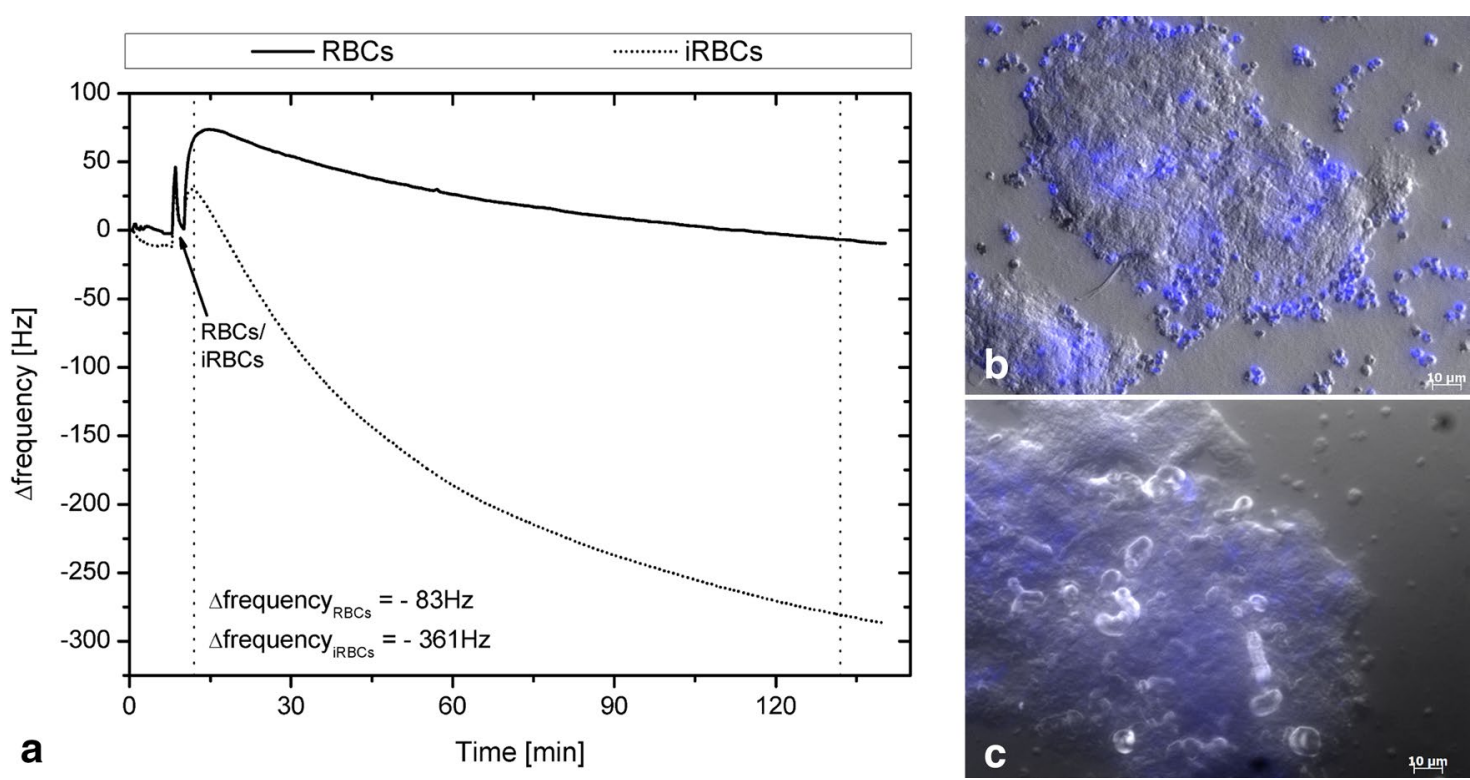

Fig. 4 Example QCM signals and pictures obtained for CD36. Measurements were carried out on the two-channel sensor platform Fidget Type 1.a The samples (iRBCs and RBCs) were injected after achievement of a stable baseline followed by a short stop-flow interval. Adhesion of iRBCs (FCR3CD36) led to a decrease of the signal. The low frequency shift obtained with RBCs shows an unspecific drift due to the alterations in the viscosity of the fluid. Frequency shifts after $2.5 \mathrm{~h}$ (time frame indicated by vertical dotted lines) are $-83 \mathrm{~Hz}$ for RBCs and $-361 \mathrm{~Hz}$ for iRBCs indicating the attachment of iRBCs to CD36 on the quartz; $\mathbf{b}$ and $\mathbf{c}$ pictures of corresponding quartzes stained with DAPI after measurement in the QCM platform confirming the results. After injection of iRBCs it can be clearly seen in (b) that iRBCs bind to the attached C32 cell membranes expressing CD36 on the quartz. In contrast in (c), after the experiment with RBCs only very few RBCs are detected on the sensor surface and only cell membranes can be seen

cells. Control experiments with human serum as unspecific analyte showed that iRBCs did not bind to serum proteins coated to PLL. The observed increase in frequency similar to the experiments of CD36 and RBCs is probably caused by a change of the visco-elasticity of the sensors surface called the missing mass effect [45]. Only by adding cells and medium to the sensor, could a conformation change of PLL be achieved so that a more rigid layer is present, influencing the sensor's behaviour. However, a control experiment with PLL and human serum shows that frequency shifts obtained with the iRBCs using the PLL/CD36- or PLL/CSA-coated sensors are due to specific interactions between the iRBCs and the receptors, and not to unspecific interactions with PLL. Parasite binding to CSA is a relevant model for placental malaria, and evaluation of inhibiting antibodies and vaccine candidates under flow conditions by QCM could complement existing methods. Further experiments with the QCM should be performed to explore the potential of this methodology in studying malaria parasites in more depth. Future experiments should include different parasite strains, including freshly isolated clinical isolates from the relevant organ (e.g., placenta), in addition experiments with inhibiting antibodies and possible blocking proteins should be performed to evaluate the potential of the methodology. One weakness of the system is that coating of a sugar as done with CSA might not represent the three-dimensional structure found in nature, a problem circumvented by coating of membranes displaying CD36 on their surface. Despite this however, this methodology has the weakness that one cannot fully control correct positioning of the membranes, and some might even be attached upside down. So far QCM technology has been applied only to analyse molecules in malaria research but not for studying the whole parasite. One group established the differentiation of Plasmodium species by analysing DNA fragments [46]. Another group evaluated the kinetics of protein interactions on the example of binding kinetics of the PfEMP1 protein var2CSA to different antibodies [47]. Here for the first time whole cell interactions of live parasites are investigated. This opens possibilities for malaria research not restricted to cytoadhesion studies. In addition, parasite invasion and egress or intracellular growth could be investigated. QCM technology is a promising tool for research in basic science on parasite biology and parasite interactions, and can also be applied for research on malaria vaccine and drug development. 

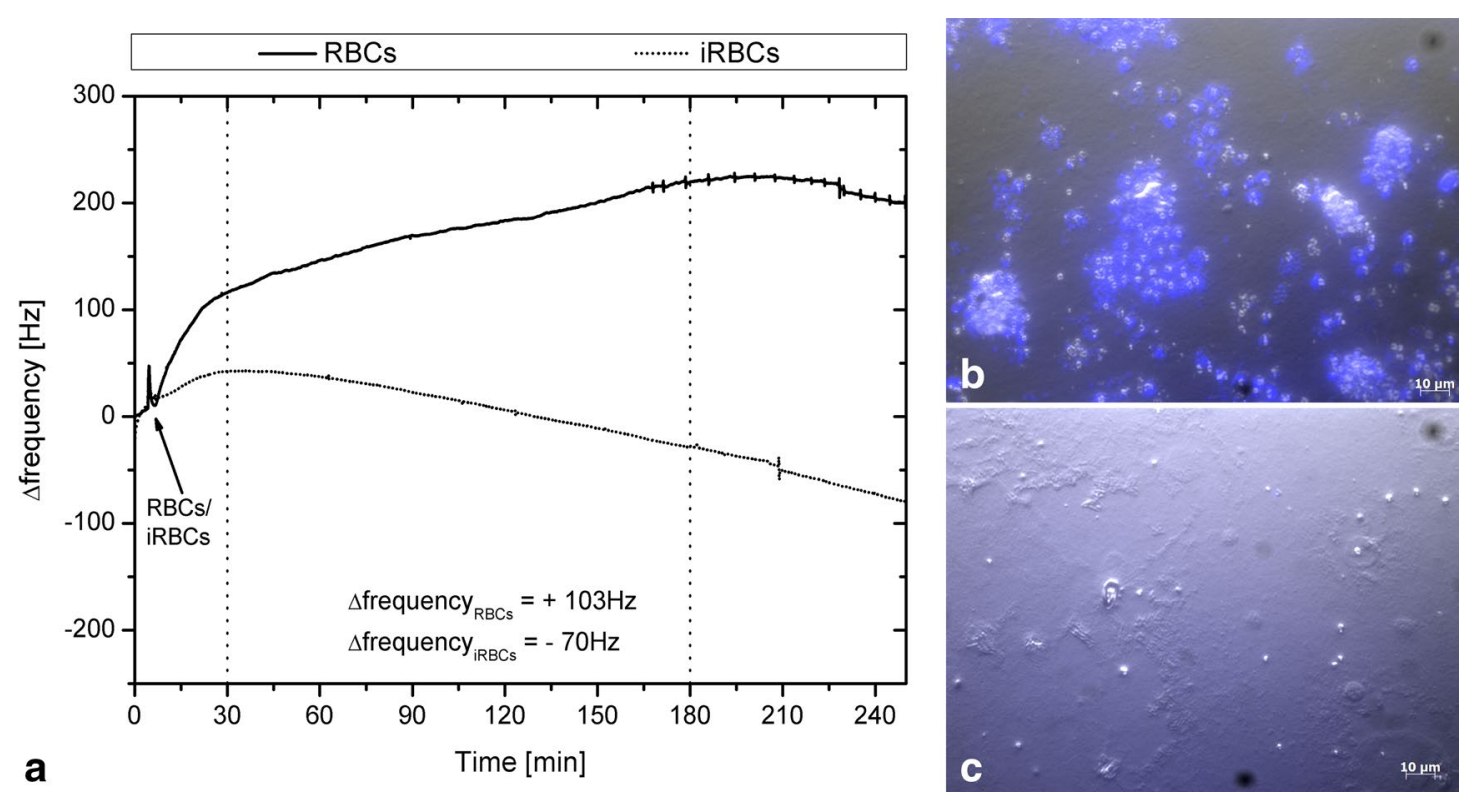

Fig. 5 Example QCM signals and pictures obtained for CSA via PLL. Measurements were carried out on the two-channel sensor platform Fidget Type 1. a Example curves show the frequency changes ( $\triangle \mathrm{f}$ ) during the course of the experiment. After injection of the samples (iRBCs and RBCs), adhesion of iRBCs (FCR3-CSA) led to a decrease of the frequency signal, whereas the frequency signal for RBCs showed no signs of attachment. Frequency shifts after $2.5 \mathrm{~h}$ (time frame indicated by vertical dotted lines) are $+47 \mathrm{~Hz}$ for RBCs and $-63 \mathrm{~Hz}$ for iRBCs indicating attachment of iRBCs to the CSA; $\mathbf{b}$ and $\mathbf{c}$ pictures of the corresponding quartzes stained with DAPI after measurement in the QCM platform confirm the results. In $\mathbf{b}$ one can see the quartz after the experiment with iRBCs showing the stained nuclei of the attached iRBCs to the CSA on the quartz. In c the quartz after the experiment with RBCs can be seen, showing only very few RBCs on the sensor surface

\section{Conclusions}

With the presented experiments QCM technology should be made accessible for malaria research. This technology enables the real-time detection of cytoadhesion of iRBCs under flow conditions without affecting viability of parasites. Head-to-head comparison to the standard assays in relevant experimental settings, for example in clinical settings within vaccine trials, are an obvious and important next step that should be taken.

\section{Additional files}

Additional file 1. Example figure of frequency shifts after adding BSA. Incubations with varying concentrations of bovine serum albumin (BSA) ( $1 \mathrm{mg} / \mathrm{ml}, 2 \%$ ) showed no additional binding events, which would be seen by a continuous drop of frequency. Therefore, no additional unspecific binding sites seem to be accessible.

Additional file 2. Example frequency shift after adding RBCs to a PLLcoated quartz. For this experiment RBCs were added to a PLL-coated quartz. It can clearly be seen that cells attached rapidly (within minutes) to the quartz indicated by a very strong frequency shift $(-3389 \mathrm{~Hz})$. This shows that PLL is unspecifically sticky to cells and RBCs attach rapidly to it.

Additional file 3. Example frequency shift of a PLL-coated quartz after adding human serum (HSA) and subsequent addition of iRBCS (FCR3CSA). For this control experiment, PLL-coated quartzes were incubated with human serum so that proteins bound to PLL. Subsequent addition of iRBCs showed no binding events (no frequency shift) as they do not bind to proteins found in the serum. This controls shows that parasites do not bind unspecifically to any analyte that is coated to PLL.

\section{Abbreviations}

ATCC: American type culture collection; BSA: bovine serum albumin; CSA: chondroitin sulfate A; IFT: indirect immunofluorescence test; iRBC: infected red blood cell; MACS: magnetic cell isolation and cells separation; NEAA: nonessential amino acid solution; PfEMP1: Plasmodium falciparum erythrocyte membrane protein 1; PLL: poly-L-lysine; QCM: quartz crystal microbalance; RBC: red blood cell; RT: room temperature.

\section{Authors' contributions}

The study was conceived and experiments designed by DK, JH, HRD, SCT, HN, BM and FKG. DK and SFM performed the experiments and DK, JH, SFM, HRD, SCT, BM and FKG analysed the data. DK, JH, SFM, HRD, SCT, HN, BM and FKG contributed reagents/materials/analysis tools and wrote the paper. All authors read and approved the final manuscript.

\section{Author details}

${ }^{1}$ Biosensor Research Group, Institute of Clinical and Experimental Transfusion Medicine, University Hospital of Tübingen, Tübingen, Germany. ${ }^{2}$ Institute of Tropical Medicine, University of Tübingen, Tübingen, Germany. ${ }^{3}$ DZIFDeutsches Zentrum für Infektionsforschung, Standort Tübingen, Germany. ${ }^{4}$ Present Address: State Health Office Baden-Württemberg, Stuttgart, Germany. ${ }^{5}$ Present Address: $3 \mathrm{~T} \mathrm{GmbH} \mathrm{\&} \mathrm{Co} \mathrm{KG,} \mathrm{Tuttlingen,} \mathrm{Germany.}$

\section{Acknowledgements}

Serum for parasite culture was kindly provided by Torsten J. Schulze, Blood Donation Centre, Institute of Transfusion Medicine and Immunology, Mannheim, Germany. We acknowledge support by Deutsche Forschungsgemeinschaft and Open Access Publishing Fund of University of Tübingen.

\section{Competing interests}

HRD and FKG work at 3t-analytic, Germany.

Received: 23 January 2016 Accepted: 4 June 2016

Published online: 13 June 2016 


\section{References}

1. Cooper MA, Singleton VT. A survey of the 2001 to 2005 quartz crystal microbalance biosensor literature: applications of acoustic physics to the analysis of biomolecular interactions. J Mol Recognit. 2007;20:154-84. doi:10.1002/jmr.826.

2. Uttenthaler E, Schraml M, Mandel J, Drost S. Ultrasensitive quartz crystal microbalance sensors for detection of M13-Phages in liquids. Biosens Bioelectron. 2001;16:735-43.

3. Speight RE, Cooper MA. A survey of the 2010 quartz crystal microbalance literature. J Mol Recognit. 2012;25:451-73. doi:10.1002/jmr.2209.

4. Ward MD, Buttry DA. In situ interfacial mass detection with piezoelectric transducers. Science. 1990;249:1000-7. doi:10.1126/science.249.4972.1000.

5. Becker B, Cooper MA. A survey of the 2006-2009 quartz crystal microbalance biosensor literature. J Mol Recognit. 2011;24:754-87. doi:10.1002/jmr.1117.

6. Saitakis M, Gizeli E. Acoustic sensors as a biophysical tool for probing cell attachment and cell/surface interactions. Cell Mol Life Sci. 2012;69:35771. doi:10.1007/s00018-011-0854-8.

7. WHO. World malaria report. Geneva: World Heath Organization; 2015.

8. Baruch DI, Gormely JA, Ma C, Howard RJ, Pasloske BL. Plasmodium falciparum erythrocyte membrane protein 1 is a parasitized erythrocyte receptor for adherence to CD36, thrombospondin, and intercellular adhesion molecule 1. Proc Natl Acad Sci USA. 1996:93:3497-502.

9. Gardner JP, Pinches RA, Roberts DJ, Newbold CI. Variant antigens and endothelial receptor adhesion in Plasmodium falciparum. Proc Natl Acad Sci USA. 1996;93:3503-8.

10. Nogueira PA, Wunderlich G, Pereira da Silva LH. Variant antigens of Plasmodium falciparum encoded by the var multigenic family are multifunctional macromolecules. Res Microbiol. 2001;152:141-7.

11. Chulay JD, Ockenhouse CF. Host receptors for malaria-infected erythrocytes. Am J Trop Med Hyg. 1990;43(2 Pt 2):6-14.

12. Beeson JG, Brown GV. Pathogenesis of Plasmodium falciparum malaria: the roles of parasite adhesion and antigenic variation. Cell Mol Life Sci. 2002:59:258-71

13. Scherf A, Pouvelle B, Buffet PA, Gysin J. Molecular mechanisms of Plasmodium falciparum placental adhesion. Cell Microbiol. 2001;3:125-31.

14. Fried M, Duffy PE. Adherence of Plasmodium falciparum to chondroitin sulfate A in the human placenta. Science. 1996;272:1502-4.

15. Cojean S, Jafari-Guemouri S, Le Bras J, Durand R. Cytoadherence characteristics to endothelial receptors ICAM-1 and CD36 of Plasmodium falciparum populations from severe and uncomplicated malaria cases. Parasite. 2008;15:163-9.

16. Barnwell JW, Asch AS, Nachman RL, Yamaya M, Aikawa M, Ingravallo P. A human 88-kD membrane glycoprotein (CD36) functions in vitro as a receptor for a cytoadherence ligand on Plasmodium falciparum-infected erythrocytes. J Clin Invest. 1989;84:765-72. doi:10.1172/JCI114234.

17. Udomsangpetch R, Taylor BJ, Looareesuwan S, White NJ, Elliott JF, Ho M. Receptor specificity of clinical Plasmodium falciparum isolates: nonadherence to cell-bound E-selectin and vascular cell adhesion molecule-1. Blood. 1996;88:2754-60.

18. Viebig NK, Levin E, Dechavanne S, Rogerson SJ, Gysin J, Smith JD, et al. Disruption of var2csa gene impairs placental malaria associated adhesion phenotype. PLoS One. 2007;2:e910. doi:10.1371/journal.pone.0000910.

19. Fried $M$, Avril M, Chaturvedi R, Fernandez P, Lograsso J, Narum D, et al. Multilaboratory approach to preclinical evaluation of vaccine immunogens for placental malaria. Infect Immun. 2013:81:487-95. doi:10.1128/IAl.01106-12.

20. Garner P, Gulmezoglu AM. Drugs for preventing malaria in pregnant women. Cochrane Database Syst Rev. 2006;4:CD000169. doi:10.1002/14651858.CD000169.pub2.

21. Steketee RW, Nahlen BL, Parise ME, Menendez C. The burden of malaria in pregnancy in malaria-endemic areas. Am J Trop Med Hyg. 2001;64(1-2 Suppl):28-35.

22. Fusai T, Parzy D, Spillmann D, Eustacchio F, Pouvelle B, Lepolard C, et al. Characterisation of the chondroitin sulphate of Saimiri brain microvascular endothelial cells involved in Plasmodium falciparum cytoadhesion. Mol Biochem Parasitol. 2000;108:25-37.

23. Turner L, Lavstsen T, Berger SS, Wang CW, Petersen JE, Avril M, et al. Severe malaria is associated with parasite binding to endothelial protein $C$ receptor. Nature. 2013:498:502-5. doi:10.1038/nature12216.

24. Paton D, Faragher B, Mustaffa KM, Szestak T, Barrett SD, Craig AG. Automated counting for Plasmodium falciparum cytoadherence experiments. Malar J. 2011;10:91. doi:10.1186/1475-2875-10-91.
25. Hempel C, Boisen IM, Efunshile A, Kurtzhals JA, Staalso T. An automated method for determining the cytoadhesion of Plasmodium falciparuminfected erythrocytes to immobilized cells. Malar J. 2015;14:112. doi:10.1186/s12936-015-0632-4.

26. Carvalho BO, Lopes SC, Nogueira PA, Orlandi PP, Bargieri DY, Blanco YC, et al. On the cytoadhesion of Plasmodium vivax-infected erythrocytes. J Infect Dis. 2010;202:638-47. doi:10.1086/654815.

27. Raventos-Suarez C, Kaul DK, Macaluso F, Nagel RL. Membrane knobs are required for the microcirculatory obstruction induced by Plasmodium falciparum-infected erythrocytes. Proc Natl Acad Sci USA. 1985;82:3829-33.

28. Nash GB, Cooke BM, Marsh K, Berendt A, Newbold C, Stuart J. Rheological analysis of the adhesive interactions of red blood cells parasitized by Plasmodium falciparum. Blood. 1992;79:798-807.

29. Herricks T, Seydel KB, Turner G, Molyneux M, Heyderman R, Taylor T, et al. A microfluidic system to study cytoadhesion of Plasmodium falciparum infected erythrocytes to primary brain microvascularendothelial cells. Lab Chip. 2011;11:2994-3000. doi:10.1039/c1lc20131j.

30. Herricks T, Avril M, Janes J, Smith JD, Rathod PK. Clonal variants of Plasmodium falciparum exhibit a narrow range of rolling velocities to host receptor CD36 under dynamic flow conditions. Eukaryot Cell. 2013;12:1490-8. doi:10.1128/EC.00148-13.

31. Cooke BM, Coppel RL. Cytoadhesion and falciparum malaria: going with the flow. Parasitol Today. 1995;11:282-7.

32. Beeson JG, Rogerson SJ, Cooke BM, Reeder JC, Chai W, Lawson AM, et al. Adhesion of Plasmodium falciparum-infected erythrocytes to hyaluronic acid in placental malaria. Nat Med. 2000;6:86-90. doi:10.1038/71582.

33. Xu X, Efremov AK, Li A, Lai L, Dao M, Lim CT, et al. Probing the cytoadherence of malaria infected red blood cells under flow. PLoS One. 2013;8:e64763. doi:10.1371/journal.pone.0064763.

34. Phiri H, Montgomery J, Molyneux M, Craig A. Competitive endothelial adhesion between Plasmodium falciparum isolates under physiological flow conditions. Malar J. 2009;8:214. doi:10.1186/1475-2875-8-214.

35. Adams Y, Rowe JA. The effect of anti-rosetting agents against malaria parasites under physiological flow conditions. PLoS One. 2013;8:e73999. doi:10.1371/journal.pone.0073999.

36. Kalantari N, Ghaffari S, Bayani M. Plasmodium falciparum: adhesion phenotype of infected erythrocytes using classical and mini-column cytoadherence techniques. Iran J Parasitol. 2013;8:158-66.

37. Trager W, Jensen JB. Human malaria parasites in continuous culture. Science. 1976;193:673-5.

38. Marsh K, Marsh VM, Brown J, Whittle HC, Greenwood BM. Plasmodium falciparum: the behavior of clinical isolates in an in vitro model of infected red blood cell sequestration. Exp Parasitol. 1988;65:202-8.

39. Ljungström I, Perlmann H, Schlichtherle M, Scherf A, Wahlgren M. Methods in Malaria Research. 4th ed. Manassas: MR4/ATCC; 2004

40. Beeson JG, Brown GV, Molyneux ME, Mhango C, Dzinjalamala F, Rogerson SJ. Plasmodium falciparum isolates from infected pregnant women and children are associated with distinct adhesive and antigenic properties. J Infect Dis. 1999;180:464-72. doi:10.1086/314899.

41. Ribaut C, Berry A, Chevalley S, Reybier K, Morlais I, Parzy D, et al. Concentration and purification by magnetic separation of the erythrocytic stages of all human Plasmodium species. Malar J. 2008;7:45. doi:10.1186/1475-2875-7-45.

42. Sinn S, Muller L, Drechsel H, Wandel M, Northoff H, Ziemer G, et al. Platelet aggregation monitoring with a newly developed quartz crystal microbalance system as an alternative to optical platelet aggregometry. Analyst. 2010;135:2930-8. doi:10.1039/c0an00474j.

43. Sinn S, Eichler M, Muller L, Bunger D, Groll J, Ziemer G, et al. NCO-sP(EOstat-PO) coatings on gold sensors - a QCM study of hemocompatibility. Sensors. 2011:11:5253-69. doi:10.3390/s110505253.

44. Gehring FK. http://www.3t-analytik.de/. Accessed 31 July 2015.

45. Voinova MV, Jonson M, Kasemo B. Missing mass effect in biosensor's QCM applications. Biosens Bioelectron. 2002;17:835-41.

46. Wangmaung N, Chomean S, Promptmas C, Mas-Oodi S, Tanyong D, Ittarat W. Silver quartz crystal microbalance for differential diagnosis of Plasmodium falciparum and Plasmodium vivax in single and mixed infection. Biosens Bioelectron. 2014;62:295-301. doi:10.1016/j.bios.2014.06.052.

47. Joergensen LM, Salanti A, Dobrilovic T, Barfod L, Hassenkam T, Theander TG, et al. The kinetics of antibody binding to Plasmodium falciparum VAR2CSA PfEMP1 antigen and modelling of PfEMP1 antigen packing on the membrane knobs. Malar J. 2010;9:100. doi:10.1186/1475-2875-9-100. 